\title{
Renal Outcomes Associated with the Use of Non-Insulin Antidiabetic Pharmacotherapy: A Review of Current Evidence and Recommendations
}

This article was published in the following Dove Press journal:

International Journal of General Medicine

\author{
Mohamed Hassan Elnaem $\mathbb{D}^{1,2}$ \\ Noha O Mansour (iD ${ }^{3}$ \\ Abdulrahman Fata Nahas ${ }^{1,2}$ \\ Mohamed A Baraka (iD) \\ Ramadan Elkalmi iD ${ }^{6,7}$ \\ Ejaz Cheema (iD) ${ }^{8}$ \\ 'Department of Pharmacy Practice, \\ Faculty of Pharmacy, International Islamic \\ University Malaysia, Kuantan, Pahang, \\ ${ }^{2}$ Quality Use of Medicines Research \\ Group, Faculty of Pharmacy, International \\ Islamic University Malaysia, Kuantan, \\ Pahang, ${ }^{3}$ Pharmacy Practice Department, \\ Faculty of Pharmacy, Mansoura University, \\ Cairo, Egypt; ${ }^{4}$ Department of Clinical \\ Pharmacy, College of Pharmacy, Al Ain \\ University, Al Ain, United Arab Emirates; \\ ${ }^{5}$ Department of Clinical Pharmacy, College \\ of Pharmacy, Al-Azhar University, Cairo, \\ Egypt; ${ }^{6}$ Department of Clinical Pharmacy \\ and Pharmacotherapeutics, Dubai Pharmacy \\ College, Dubai, United Arab Emirates; \\ ${ }^{7}$ Department of Pharmacology, Faculty of \\ Medicine, Sebha University, Sabha, Libya; \\ ${ }^{8}$ School of Pharmacy, University of \\ Birmingham, Birmingham BI5 2TT, UK
}

Correspondence: Ejaz Cheema School of Pharmacy, University of Birmingham, Edgbaston, Birmingham BI5 2TT, UK

Email E.Cheema@bham.ac.uk
Background: This study aims to discuss, summarize and compare the renal outcomes associated with non-insulin antidiabetic (AD) pharmacotherapy prescribed for patients with type 2 diabetes mellitus (T2DM).

Methods: A systematic search using predefined search terms in three scholarly databases, ScienceDirect, Google Scholar, and PubMed, was conducted. Original research articles published in the English language between 2012 and 2020 that reported renal outcomes associated with the use of non-insulin AD pharmacotherapy were eligible for inclusion. Review articles, meta-analysis studies, and conference proceedings were excluded. A studyspecific data extraction form was designed to extract the author's name, country, publication year, study design, study population, objectives, key findings, and conclusions. A narrative review of the key findings that focused on renal outcomes and renal safety issues was conducted.

Results: Of the 18,872 results identified through the initial search, a total of 32 articles were included in this review. Of these, 18 of the included articles reported the renal outcomes of newer antidiabetic medications, eg, SGLT2 inhibitors and GLP-1 agonists. Eight studies focussed on the well-established antidiabetic medications, eg, metformin and sulphonylureas. The review reported three main types of the clinical impact of the prescribed AD on the renal outcomes: "renoprotective effects", "no additional risk" and "associated with a decline in renal parameters". Seventeen studies reported the renoprotective effects of $A D$, including SGLT2i studies ( $n=8)$, GLP1 studies $(n=6)$, and DPP4i studies $(n=3)$. The reported renoprotective effects included slowing down the GFR decline, improving albuminuria, and reducing renal adverse events. The "no additional risk" impact was reported in eight studies, including DPP4i studies $(n=3)$, two SGLT2i studies $(n=2)$, metformin studies $(n=2)$, and one study involving pioglitazone. Furthermore, seven studies highlighted the "associated with a decline in renal parameters" effect. Of these, three involved SGLT2i, two with metformin, and one for each DPP4i and sulphonylurea.

Conclusion: More than half of the studies included in this review supported the renoprotective effects associated with the use of AD medications, particularly GLP-1A, SGLT2i, and some of the DPP4i. Further studies involving patients with various stages of chronic kidney disease $(\mathrm{CKD})$ are required to compare AD medications' renal effects, particularly the newer agents.

Keywords: hypoglycemic agents, antidiabetic drugs, type 2 diabetes mellitus, renal effects, renal outcomes 


\section{Introduction}

Diabetes is a major public health disease that is increasingly becoming highly prevalent worldwide. The number of diabetic patients increased from 108 million to 422 million in the duration from 1980 to $2014 .^{1}$ Microvascular and macrovascular complications are common in diabetic patients. Diabetic kidney disease (DKD), also known as diabetic nephropathy, is a major microvascular complication that occurs in $20-40 \%$ of diabetic patients. ${ }^{2}$ Screening for diabetic nephropathy is typically recommended 5 years after diagnosis in type 1 diabetes and should be considered at the time of diagnosis in type 2 diabetes. ${ }^{3}$ DKD can progress to end-stage renal disease (ESRD) requiring dialysis or kidney transplantation. In diabetic patients, the development of DKD raises the risk of mortality by at least fivefold compared to patients with normal renal function. In those who develop ESRD, the risk of death is increased up to 100 -fold. This increased risk occurs due to higher rates of both cardiovascular and noncardiovascular events. ${ }^{4}$

Diabetic nephropathy is clinically diagnosed based on the presence of albuminuria and/or reduced eGFR to $<60$ $\mathrm{mL} / \mathrm{min} / 1.73 \mathrm{~m}^{2}$ in the absence of signs or symptoms of other primary causes of kidney damage. Screening for albuminuria can be performed by urinary albumin-tocreatinine ratio (UACR) in a random spot urine collection. ${ }^{5}$ Albumin excretion rate higher than $30 \mathrm{mg}$ per day or albumin/creatinine ratio (ACR) higher than 3 $\mathrm{mg} / \mathrm{mmol}$ has been established as the marker for detecting kidney damage. ${ }^{6}$ The presence of albuminuria has been identified as a risk factor for further cardiovascular complications among patients with or without diabetes. ${ }^{7}$ Albuminuria and eGFR should be monitored regularly in diabetic patients to enable timely diagnosis and monitor the progression of DKD.

Intensive glycemic control has been shown in large randomized clinical trials to delay the onset and progression of DKD in diabetic patients. ${ }^{8}$ The choice of antidiabetic regimen for patients with type 2 diabetes mellitus (T2DM) relies on an interplay of patient characteristics, severity of hyperglycemia, and available therapeutic options. Pharmacological interventions used to improve glucose control include both oral and injectables. Pharmacotherapy is typically introduced using a stepwise approach starting with oral agents, followed by injectables such as glucagon-like peptide-1 analogs (GLP-1A) and insulin. ${ }^{5}$ Commonly prescribed classes of antidiabetic
(AD) medications include biguanides, thiazolidinediones, second-generation sulphonylureas. Several drugs have been introduced over the last 15 years to treat diabetes, including sodium-glucose cotransporter-2 inhibitors, dipeptidyl peptidase-4 inhibitors, and GLP-1 analogs. Interestingly, outcomes of recent trials have indicated that these drugs may directly improve renal function beyond changes in glycaemic control. ${ }^{4}$ This review, therefore, aims to discuss, summarize, and compare the renal outcomes associated with non-insulin antidiabetic (AD) pharmacotherapy prescribed for patients with T2DM.

\section{Methods}

\section{Search Strategy and Identification of the Studies}

Three scholarly databases, ScienceDirect, Google Scholar, and PubMed, were systematically searched to identify potentially relevant studies. The search terms used were "antidiabetic drugs OR antidiabetic medications AND renal outcomes OR kidney outcomes" or "hypoglycemic agents OR hypoglycemic drugs AND adherence OR compliance". Individual names of agents and drug classes were considered to identify the potentially eligible articles. The abstracts of the eligible studies were screened for eligibility. Original research articles published between 2012 and 2020 in English and reported renal outcomes associated with the use of non-insulin antidiabetic drugs were eligible for inclusion. The searches were limited to the last eight years to provide a review of the most recently published evidence by incorporating all the relevant studies that have been published within this study period. Only studies that assessed the renal outcomes by quantitative clinical measures were eligible for inclusion. Review articles, metaanalysis, book chapters, and conference proceedings were excluded. Details for the inclusion criteria are presented in Table 1.

\section{Study Selection and Data Extraction}

Two independent reviewers reviewed the studies at the title and abstract level following the removal of duplicates. Eligible studies were identified and retrieved at full-text to consider them for inclusion. A series of meetings, in the beginning, at the middle, and end of the data extraction process were conducted to ensure consistency. The inclusion of a third reviewer was made to resolve any differences. Data were extracted from the selected studies using study-specific 
Table I Study Inclusion Criteria

\begin{tabular}{|l|l|l|}
\hline No. & Category & Inclusion Criteria \\
\hline I & $\begin{array}{l}\text { Language of } \\
\text { Publication } \\
2\end{array}$ & English \\
3 & $\begin{array}{l}\text { Publication Type } \\
\text { Outcomes }\end{array}$ & $\begin{array}{l}\text { Full-text original research article discussing the effects of AD medication on the renal system. } \\
\text { Measuring patients' renal outcomes, either primary or secondary outcome measures. }\end{array}$ \\
5 & $\begin{array}{l}\text { Methodology } \\
6\end{array}$ & $\begin{array}{l}\text { Clinical studies reported the renal outcomes of using non-insulin antidiabetic pharmacotherapy, either observational } \\
\text { or experimental. } \\
\text { Adult patients with T2DM. }\end{array}$ \\
\hline
\end{tabular}

data extraction form to identify the author's name, country, year, study design, study population, length of follow-up, objectives, key findings, and conclusions. The relevant studies were grouped according to their AD medication classification. Reporting the key findings was carried out consistently, focusing on renal outcomes and renal safety issues. For articles that reported further analyses of the previously published clinical trials, the authors included the specific part of the findings related to renal outcome assessment. The flow chart describing the selection of the articles is illustrated in Figure 1.

\section{Results}

Of the 18,872 results identified through the initial search, a total of 32 articles were included in the review. There was a variation in the number of articles discussing the renal outcomes among various antidiabetic classes. Of these, 18 of the included articles reported the renal outcomes of newer antidiabetic medications, eg, SGLT2 inhibitors and GLP-1 agonists. Eight studies focussed on the well-established antidiabetic medications, eg, metformin and sulphonylureas. The included studies were observational, randomized controlled trials, and post hoc or secondary analyses.

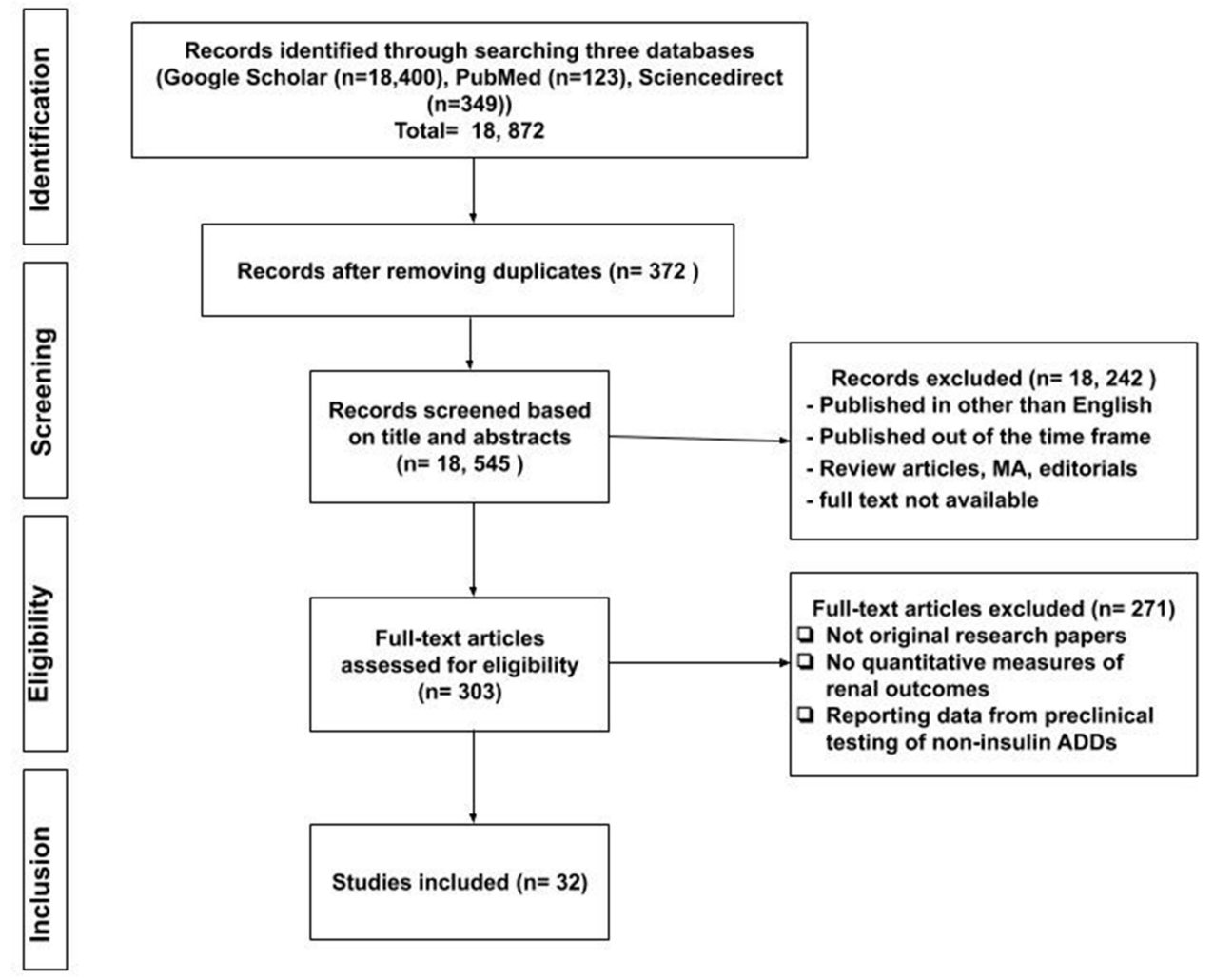

Figure I Flow chart on the selection of literature. 
The review reported three main types of the clinical impact of the prescribed AD drugs on the renal outcomes. These included the "renoprotective effects", "no additional risk, and "associated with a decline in renal parameters". Seventeen studies reported the renoprotective effects of $\mathrm{AD}$, including SGLT2 $\mathrm{i}$ studies $(\mathrm{n}=8)$, GLP1 studies $(n=6)$, and DPP4i studies $(n=3)$. The reported renoprotective effects included slowing down the GFR decline, improving albuminuria, and reducing renal adverse events. The "no additional risk" impact was reported in eight studies, including DPP4i studies ( $\mathrm{n}=3$ ), two SGLT2i studies $(\mathrm{n}=2)$, metformin studies $(\mathrm{n}=2)$, and one study involving pioglitazone. Furthermore, seven studies highlighted the "associated with a decline in renal parameters" effect. Of these, three involved SGLT2i, two with metformin, and one for each DPP4i and sulphonylurea. The overall summary of the main characteristics and findings of the included studies have been presented in Table 2

\section{Discussion}

More than half of the studies included in this review supported the renoprotective effects associated with the use of antidiabetic medications, particularly GLP-1A, SGLT2i, and some of the DPP4i group. The demonstrated renoprotective effects included slowing down the GFR decline, improving albuminuria, and reducing renal adverse events. The remaining studies either showed no additional risk or minor and temporary decline in the renal function parameters. In general, no study reported the detrimental impact of antidiabetic medications on renal outcomes that outweighed any clinical benefit.

\section{Metformin}

Metformin is the first-choice antidiabetic medication for most of the cases, and it acts as insulin sensitizers. In our review, we found that diabetic patients with eGFR 45-60 $\mathrm{mL} / \mathrm{min} / 1.73 \mathrm{~m}^{2}$ were reported to experience a lower risk of all-cause mortality with metformin administration. ${ }^{11}$ Glomerular filtration rate is considered a good indicator in assessing the functionality of the renal system. In a large cohort study ( $\mathrm{n}=124720)$, Christiansen et al reported that diabetic patients started on Metformin reported a lower risk of experiencing a severe decline of eGFR; however, the mild and moderate decline was common in those patients. $^{10}$

There is conflicting evidence that suggests that acute kidney injury (AKI) is precipitated by metformin-associated lactic acidosis. Mariano et al reported that the use of Metformin was associated with the occurrence of lactic acidosis, resulting in AKI that required renal replacement therapy (RRT). ${ }^{12}$ However, the study reported a high survival rate following RRT. Moreover, most of the patients in this study experienced AKI when they had dehydration, shock, or oliguria. These conditions are previously known to result in increased Metformin levels, which has been proposed to precipitate lactic acidosis. ${ }^{41}$

On the contrary, an observational study involving 25,148 patients found no significant association of Metformin uses with an increased risk for AKI in terms of lactic acidosis. ${ }^{9}$ It is important to note that Mariano et al (2017) study was retrospective, which focused on the patients' survival rate, while Bell et al (2017) study focused more on the relationship between consumption of Metformin and the occurrence of AKI due to lactic acidosis. Further studies are required to explore the risk of Metformin for AKI in terms of lactic acidosis.

\section{Sodium-Glucose Co-Transporter-2 Inhibitors (SGLT2i)}

Sodium-glucose co-transporter-2 (SGLT2) inhibitors act by inhibiting the SGLT2 protein in the proximal convoluted tubule, stopping the reuptake of both sodium and glucose. The consequent increased urinary excretion of glucose improves glycemic control and weight loss, and the decreased reabsorption of sodium and glucose in the tubules promotes natriuresis and reducing blood pressure.${ }^{42}$ In this review, more than half of the included studies involving SGLT2 inhibitors reported positive renal protective outcomes of SGLT2 inhibitors in patients. Three large multinational, randomized trials that were primarily designed to evaluate the cardiovascular outcomes of dapagliflozin, canagliflozin, and empagliflozin reported the renal endpoints as secondary outcomes. The strongest evidence emerged from the DECLARE-TIMI 58 trial, which secondarily investigated the renal effects of dapagliflozin in diabetic patients with existing cardiovascular disease or at high risk for cardiovascular disease (n $=17160$ ). In participants using dapagliflozin, there was a reduced risk of a sustained decline in eGFR, and the risk of ESRD or renal death. ${ }^{14}$ This positive nephroprotective role was reproduced in a similar patient population for canagliflozin from the CANVAS Program study $(n=10142)$ and empagliflozin from a secondary analysis of the EMPA-REG OUTCOME study among Asian patients $(n=1517) .{ }^{17,23}$ Although these are strong positive 


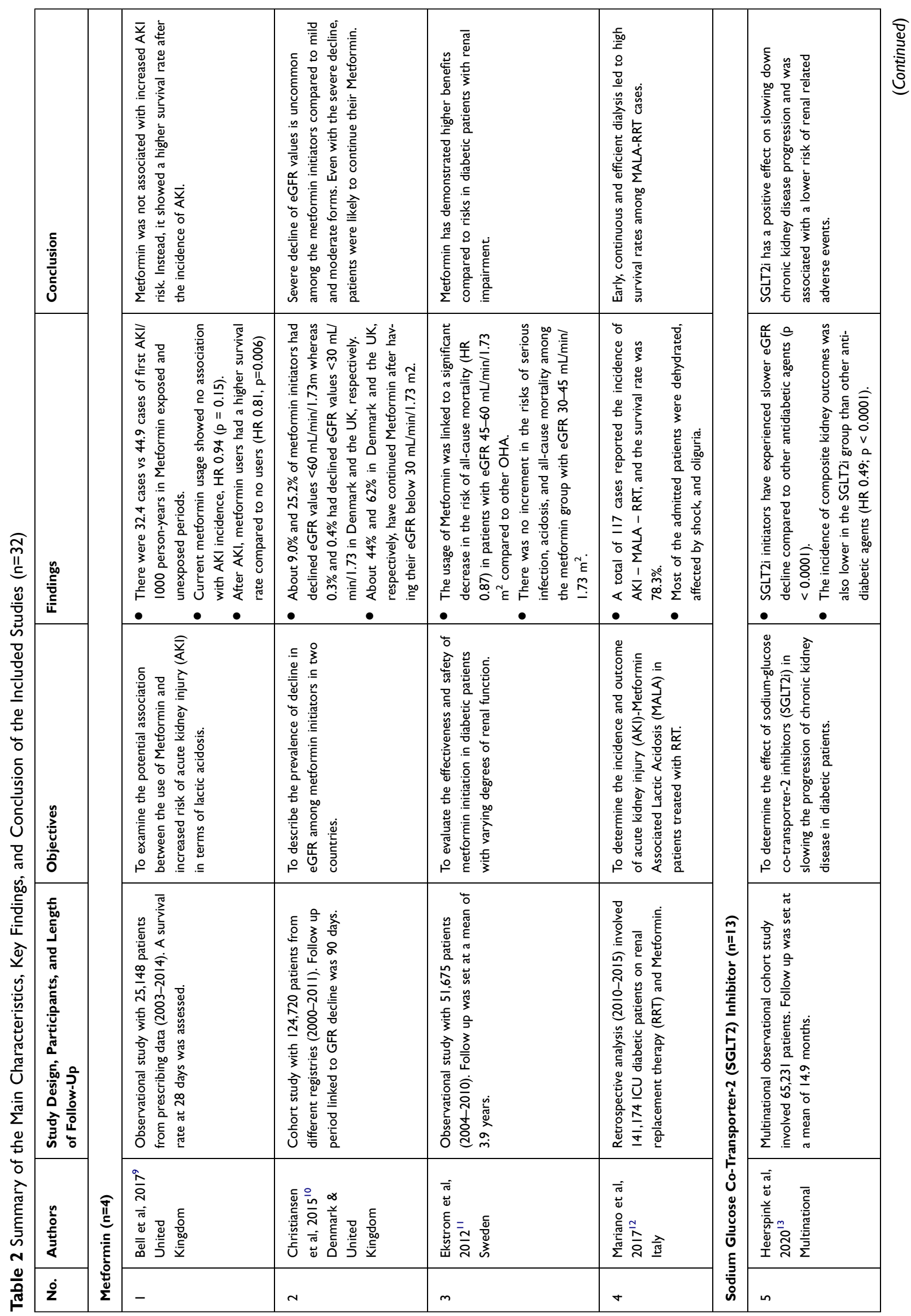




\begin{tabular}{|c|c|c|c|c|c|}
\hline 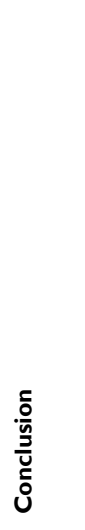 & 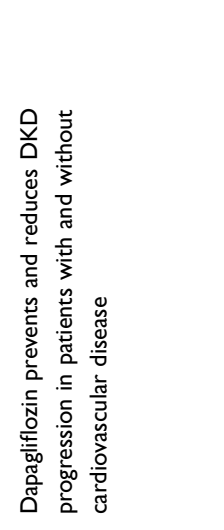 & 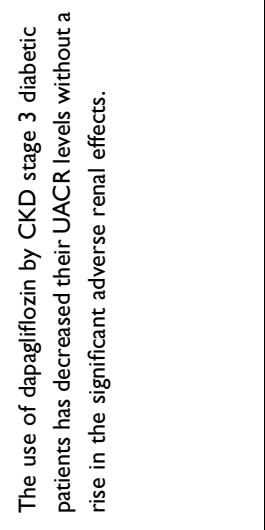 & 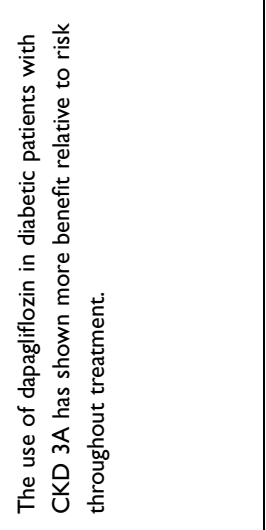 & 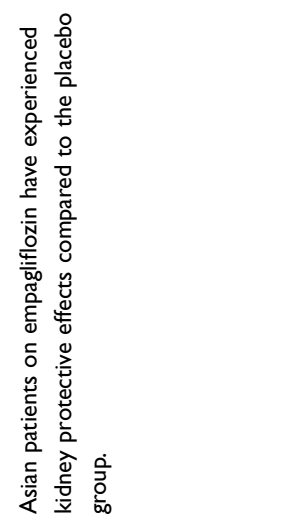 & 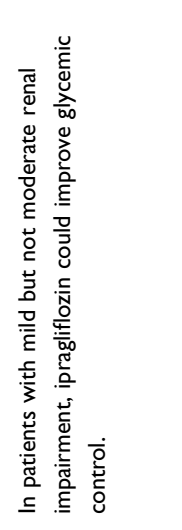 \\
\hline 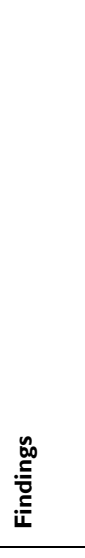 & 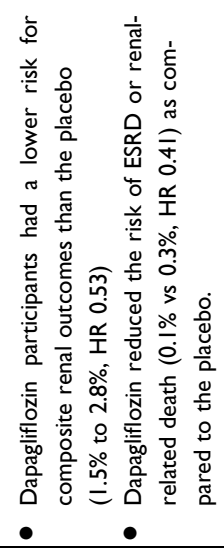 & 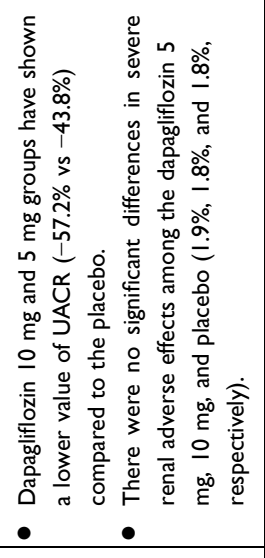 & 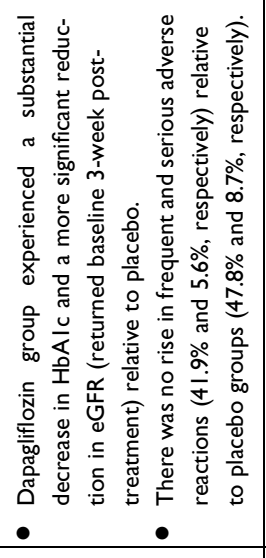 & 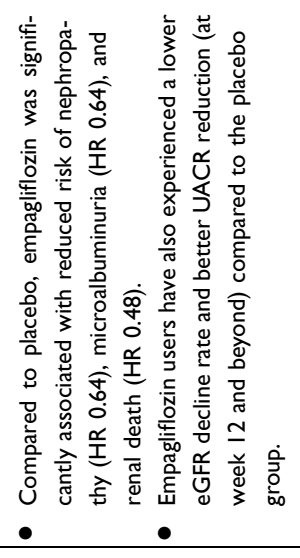 & 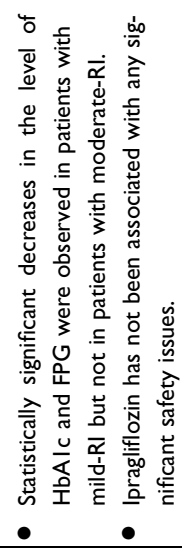 \\
\hline 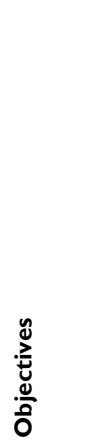 & 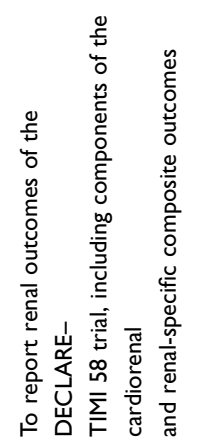 & 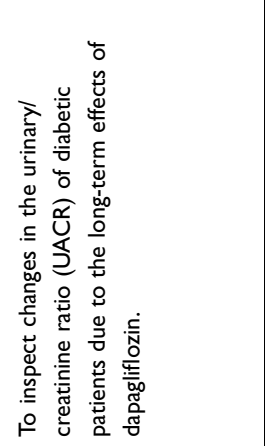 & 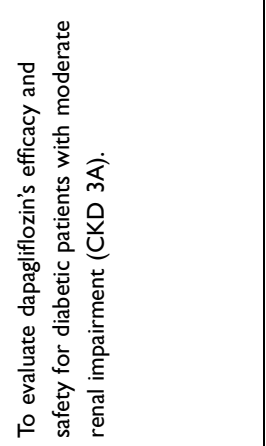 & 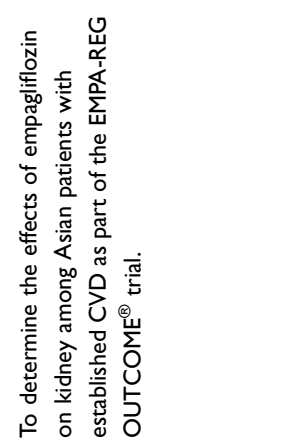 & 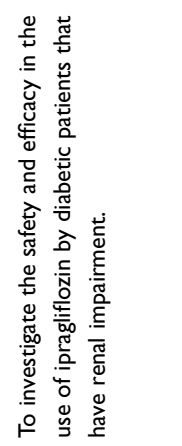 \\
\hline 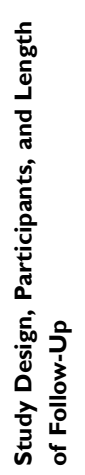 & 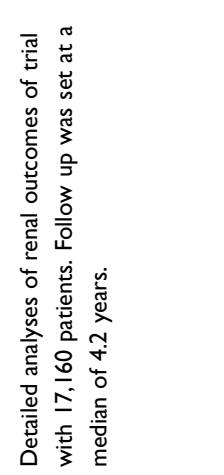 & 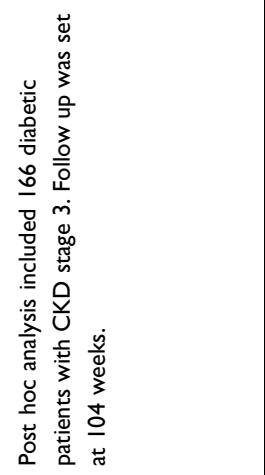 & 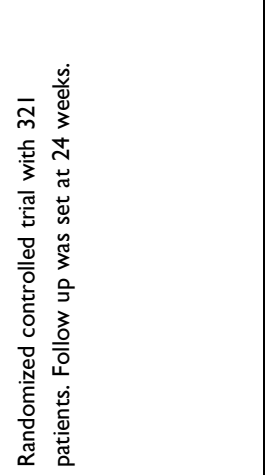 & 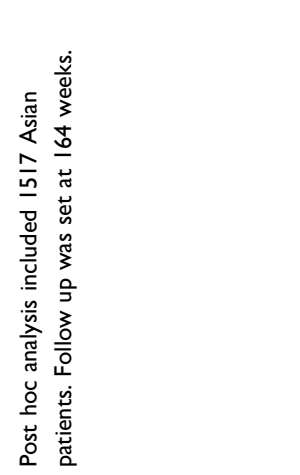 & 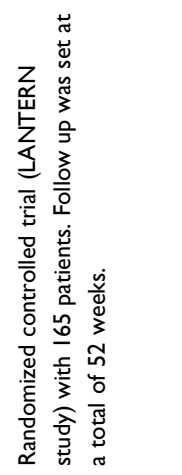 \\
\hline $\begin{array}{l}\frac{n}{0} \\
\frac{\dot{p}}{z} \\
\frac{z}{z}\end{array}$ & 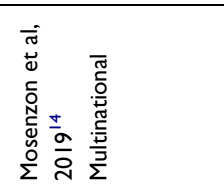 & 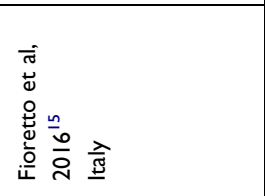 & 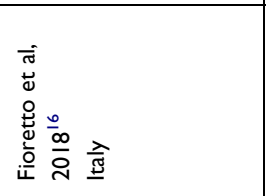 & 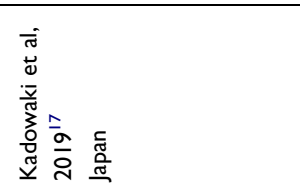 & 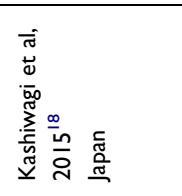 \\
\hline$\dot{\mathbf{z}}$ & $\circ$ & n & $\infty$ & $\alpha$ & 으 \\
\hline
\end{tabular}




\begin{tabular}{|c|c|c|c|c|c|}
\hline 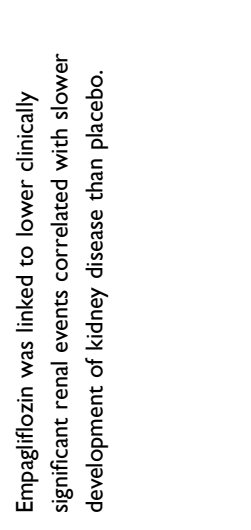 & 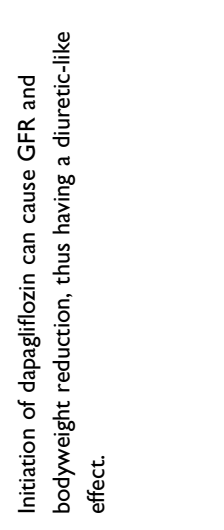 & 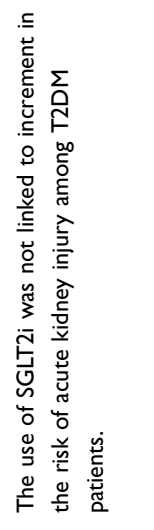 & 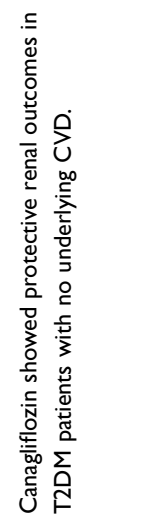 & 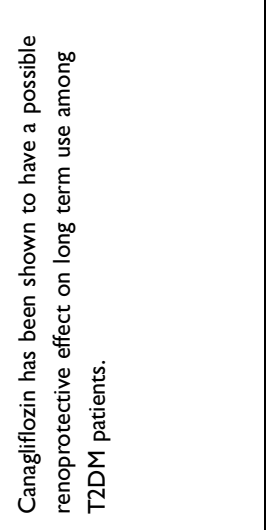 & 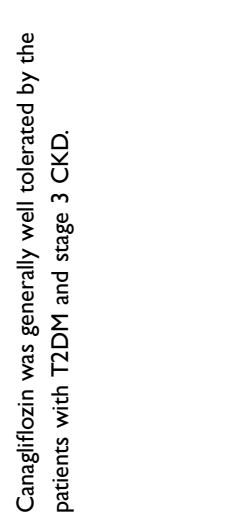 \\
\hline 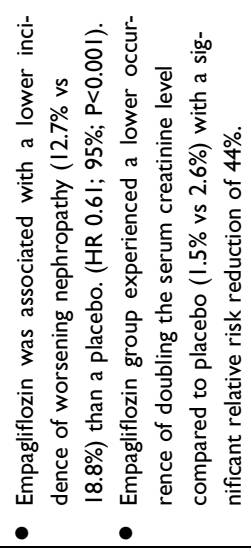 & 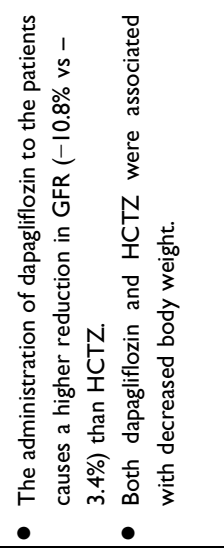 & 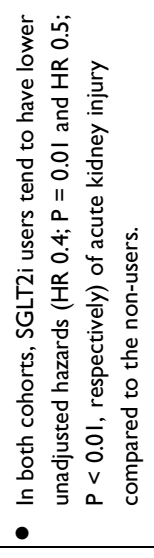 & 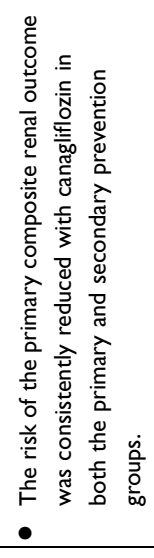 & 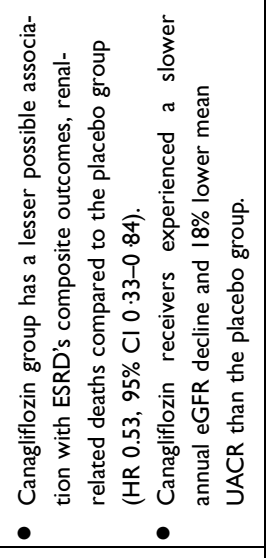 & 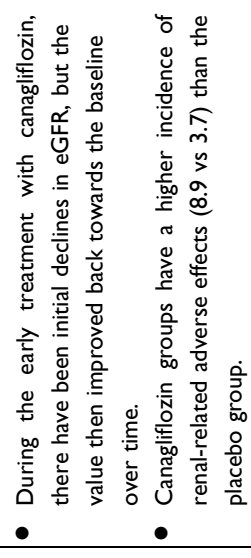 \\
\hline 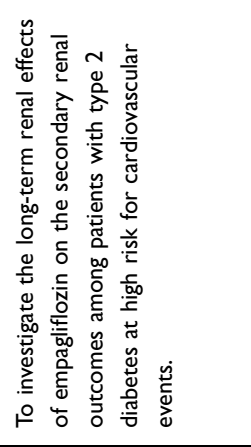 & 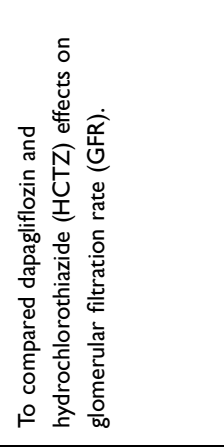 & 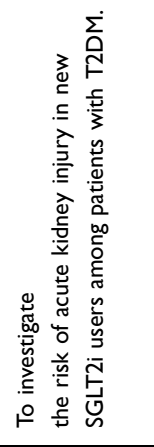 & 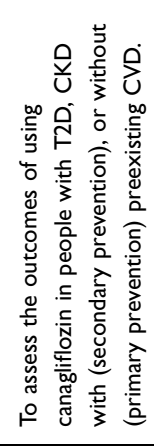 & 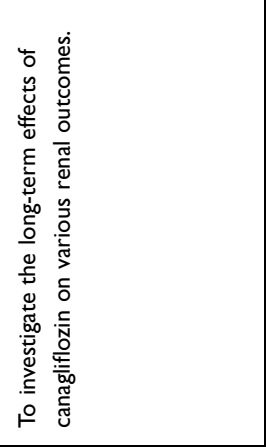 & 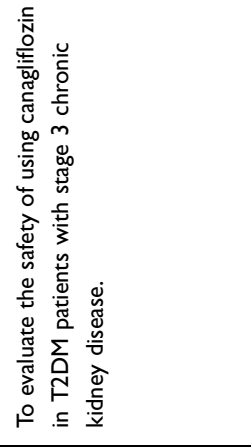 \\
\hline 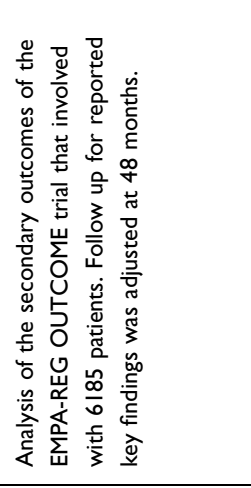 & 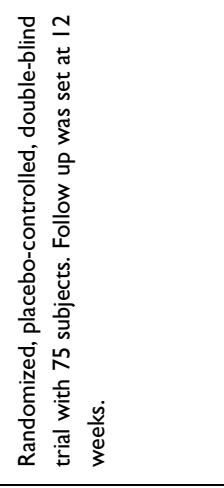 & 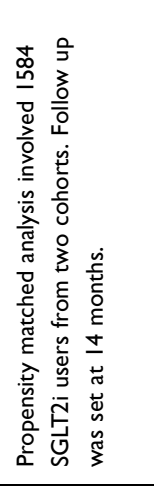 & 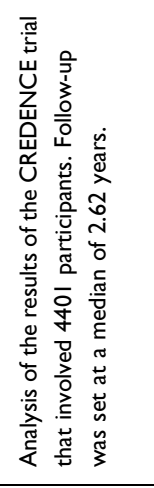 & 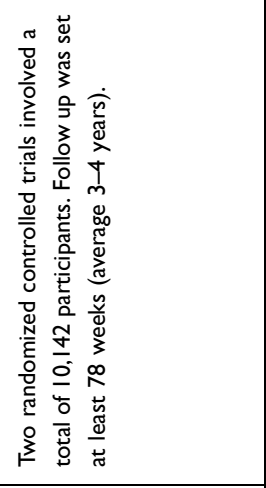 & 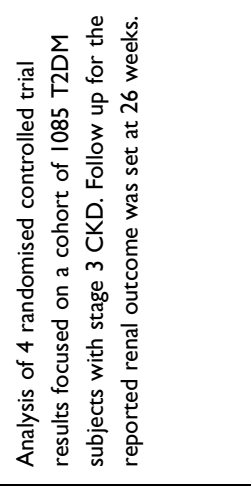 \\
\hline 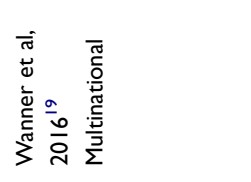 & 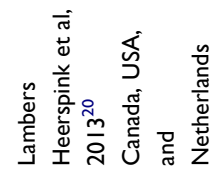 & 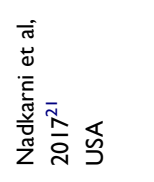 & 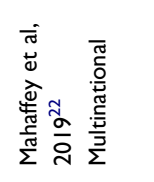 & 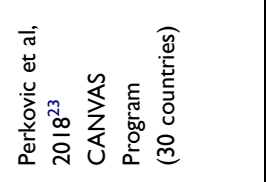 & 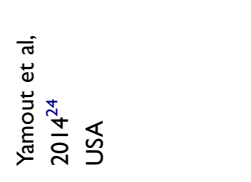 \\
\hline$=$ & $\simeq$ & $\underline{m}$ & $\underline{ \pm}$ & $\underline{\underline{n}}$ & 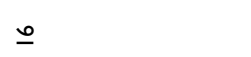 \\
\hline
\end{tabular}




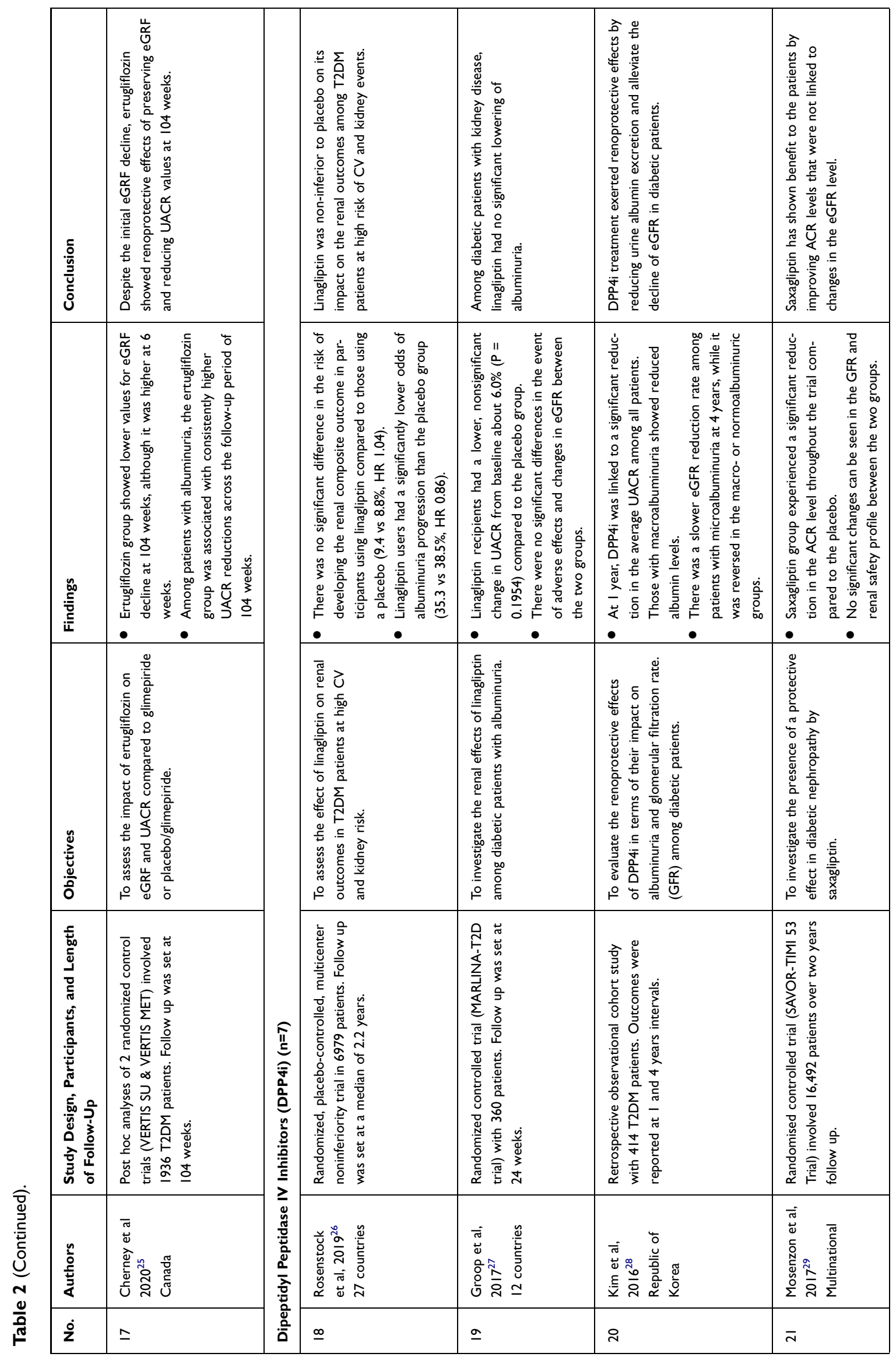




\begin{tabular}{|c|c|c|c|c|c|c|}
\hline 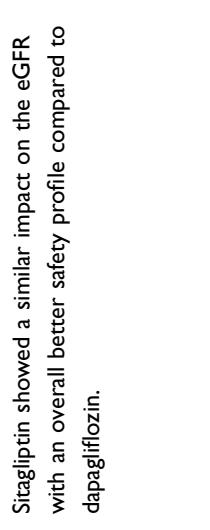 & 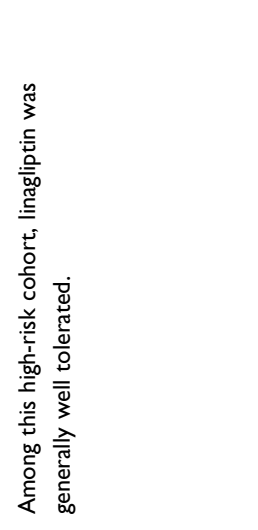 & 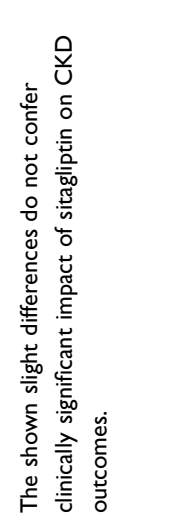 & & 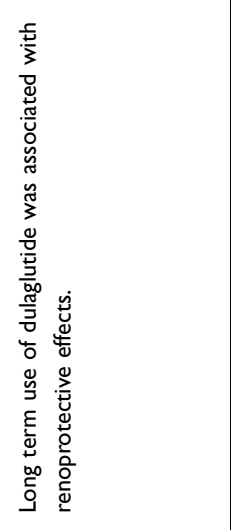 & 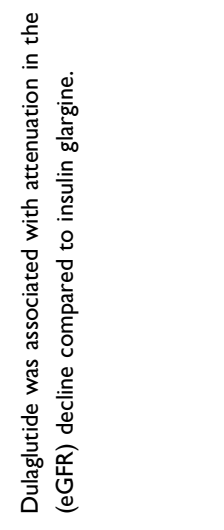 & 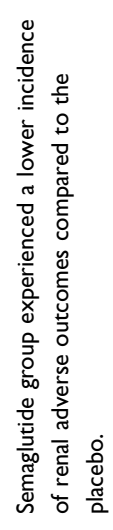 \\
\hline 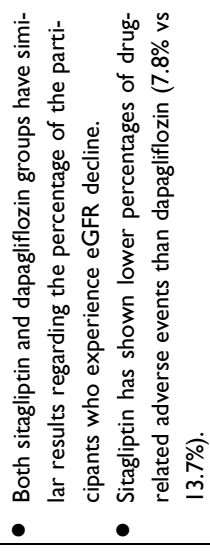 & 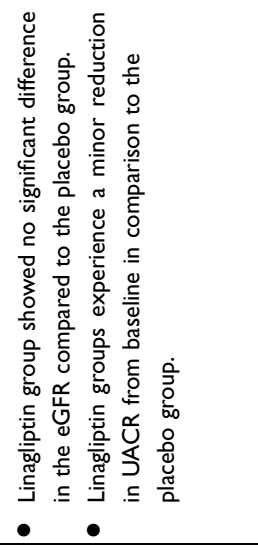 & 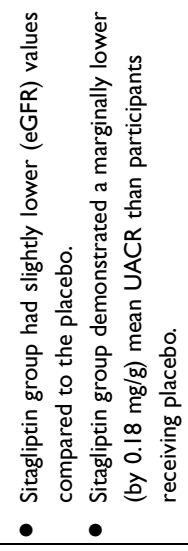 & & 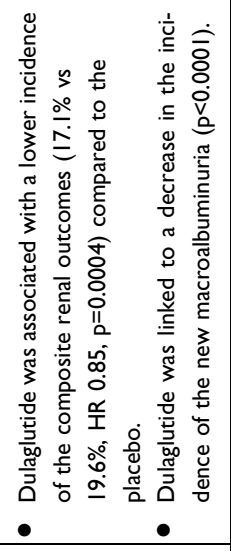 & 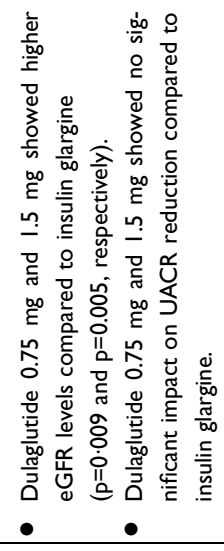 & 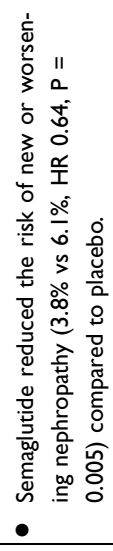 \\
\hline 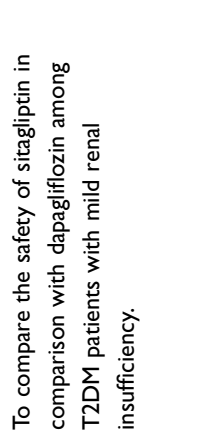 & 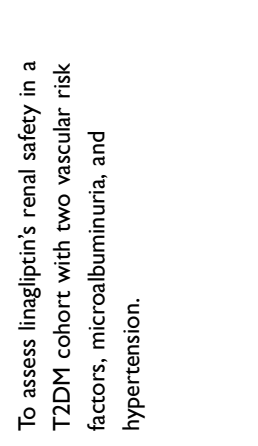 & 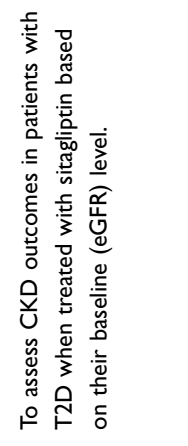 & & 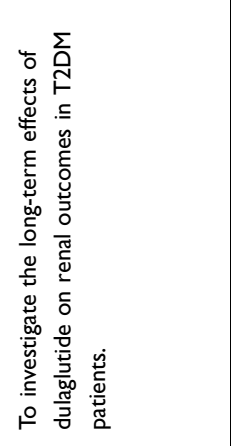 & 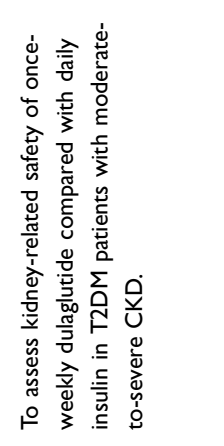 & 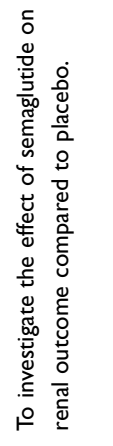 \\
\hline 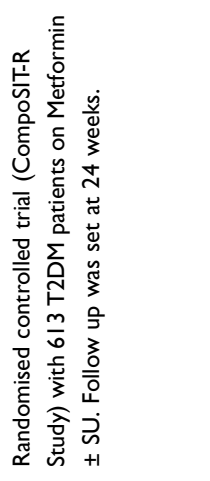 & 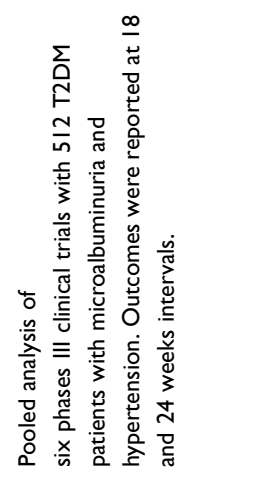 & 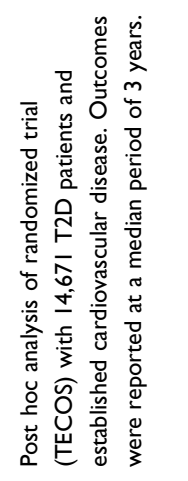 & 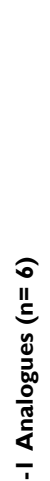 & 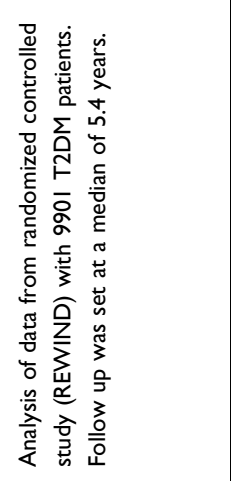 & 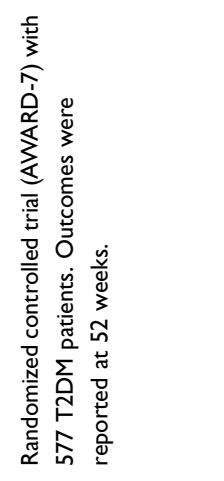 & 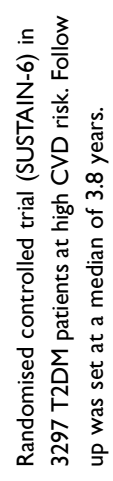 \\
\hline 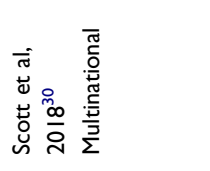 & 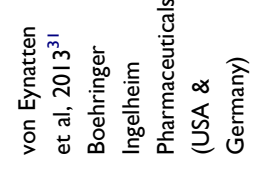 & 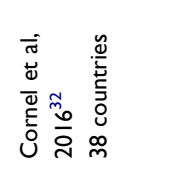 & 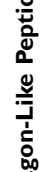 & 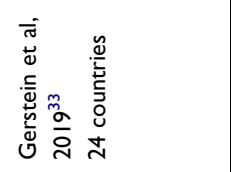 & 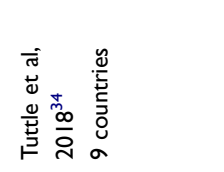 & 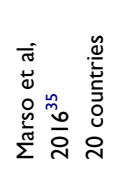 \\
\hline$\pi$ & $\tilde{\sim}$ & $\stackrel{ \pm}{\sim}$ & $\bar{\sigma}$ & $\stackrel{\sim}{\sim}$ & $\stackrel{\sim}{\sim}$ & $\hat{\lambda}$ \\
\hline
\end{tabular}




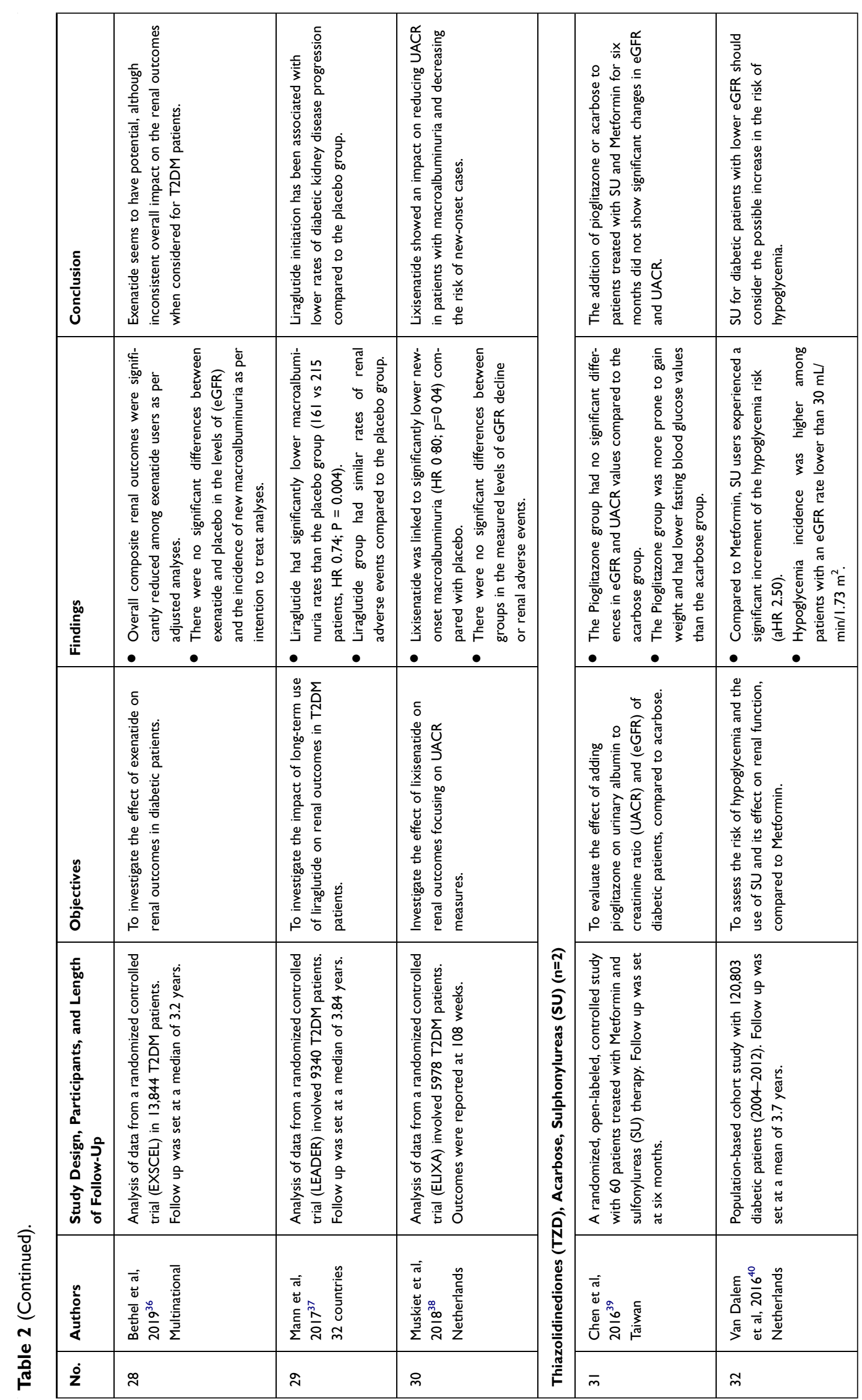


evidence points, a point to consider is the varying definitions of the renal outcomes utilized in these trials. Apart from the cardiovascular safety trials, the CREDENCE trial $(\mathrm{n}=4401)$ was primarily designed to assess canagliflozin's renal safety in diabetic patients with preexisting renal disease..$^{22}$ It reported reductions in the proportions of participants using canagliflozin who attained ESRD, RRT, or renal death. On the other hand, some studies showed a reduction in the renal parameters, either GFR or UACR, although these effects tended to be minor and reversible in many cases. ${ }^{15,20,24}$

To explain the positive renal outcomes of SGLT2 inhibitors in diabetic patients, it is reasonable to say that SGLT2 inhibitors promote nephroprotection by direct and indirect mechanisms. The direct mechanisms involve osmotic diuresis in overloaded cases in synergy with the given diuretic therapy and the suppression of oxidative stress and inflammation pathways. ${ }^{43}$ Meanwhile, the indirect mechanisms include the ability to reduce blood pressure, body fat, body weight in addition to the uricosuric effect. ${ }^{43}$ In a clinical trial, dapagliflozin was found to reduce blood pressure and body weight near the values reported for hydrochlorothiazide, although different underlying mechanisms exert diuretic effects. ${ }^{20}$ As a consequence of reduced-sodium and glucose reabsorption in the tubules, SGLT2i enhances sodium and chloride delivery to the macula densa, resulting in the increased local secretion of vasoconstrictors that decrease the intraglomerular pressure and decrease the risk of hyperfiltration. ${ }^{44}$ SGLT2i showed a remarkable nephroprotective effect by reversing the afferent arteriole's vasodilatation to reduce the albumin excretion. ${ }^{44}$ Therefore, SGLT2i has been shown to mitigate two of the early pathophysiological mechanisms of DKD, hyperfiltration, and glomerular hypertension. The main precautions related to the use of SGLT2i over different eGFR values should also be emphasized where overall it should not be started at $<60$ and to be discontinued when it is below $45 \mathrm{~mL} / \mathrm{min} / 1.73 \mathrm{~m} 2$ taking into account the minimum glycemic impact at this level. ${ }^{44}$ Recent evidence suggest to consider SGLT2i treatment if eGFR is 30 to $<90 \mathrm{~mL} / \mathrm{min} / 1.73 \mathrm{~m} 2 .{ }^{45}$ A recent real-world data analysis has highlighted that although SGLT2i use was not linked to an increase in AKI's risk, increased reduction in eGFR was observed among patients with relatively reduced eGFR levels. ${ }^{46}$ As discussed with cardiovascular outcomes, ${ }^{47}$ it seems that relatively more evidence supports empagliflozin compared to other SGLT2i such as dapagliflozin and canagliflozin. ${ }^{44}$

\section{Dipeptidyl Peptidase 4 Inhibitors (Dpp4i)}

Dipeptidyl peptidase IV (DPP4) inhibitors, also known as gliptins, act by several mechanisms: slowing down the gastric emptying and reducing postprandial glucagon. In our review, we included seven articles for this class of AD medication. Three studies reported positive outcomes associated with DPP4 inhibitors, while the remaining four studies showed no additional risk $(\mathrm{n}=3)$ or insignificant difference in eGFR decline $(n=1)$ with the use of DPP4 inhibitors. A cohort study that assessed several types of DPP4 inhibitors among diabetic patients had identified a reduction in average ACR in macroalbuminuric patients while in microalbuminuria, the rate of eGFR reduction was alleviated. ${ }^{28}$ Patients taking linagliptin in two trials have shown no significant change in eGFR, proving that there was neither improvement nor exacerbation of the condition. ${ }^{27,31}$ However, Rosenstock et al reported a smaller risk for albuminuria progression in diabetic patients using linagliptin compared to those on placebo. ${ }^{26}$ Although there were no significant changes in eGFR of diabetic patients with saxagliptin, Mosenzon et al reported a reduction in the ACR in their trial $(n=16492) .{ }^{29}$ A randomized controlled trial that administered sitagliptin to the patients also reported that the sitagliptin group was found to have a lower incidence of adverse events than dapagliflozin. ${ }^{30}$ Another randomized trial supported the safety of sitagliptin use among CKD patients, which reported no clinically significant effect of sitagliptin on CKD progression among 14,671 T2D patients. ${ }^{32}$

DPP4i plays a significant role in glucose metabolism. It rapidly degrades GLP-1, an incretin hormone secreted postprandially, which stimulates the pancreatic GLP-1 receptor. This stimulation results in insulin secretion from $\beta$-cells and also inhibits glucagon secretion from $\alpha$ cells. ${ }^{48}$ DPP4 exists in several tissues, although its highest level is found in the kidneys, increasing diabetic nephropathy. Studies revealed that the use of DPP4i resulted in a nephroprotective effect via glycemic control. Additionally, the inhibition of DPP4 showed a renoprotective effect through other pathways such as reducing oxidative stress, inflammation and ameliorating renal fibrosis. ${ }^{49}$ Moreover, the use of DPP4 inhibitors in T2D patients induced natriuresis, ${ }^{50}$ which might positively affect renal parenchymal hypertension. ${ }^{51}$ In general, most of the findings from the reviewed articles support the positive effects of DPP4i on albuminuria and reduce ACR in T2D patients. 


\section{Glucagon-Like Peptide-I Analogues (GLP-} IA)

Although only six of the included studies involved GLP$1 \mathrm{~A}$, all these studies showed potential renoprotective effects of GLP-1A. Liraglutide, an example of GLP-1A that acts by stimulating the release of insulin into the circulation, increasing urinary sodium excretion and decreasing urinary albumin excretion, has been reported to maintain the impact on reducing macroalbuminuria over approximately four years in a secondary analysis of the data of LEADER trial. ${ }^{37}$ Considering that macroalbuminuria is linked to the incidence of cardiovascular events, so this could be one of the possible mechanisms for GLP-1A to show desirable cardiovascular outcomes. ${ }^{7,52}$ As discussed in cardiovascular outcome trials, it seems that relatively more strong evidence supports liraglutide than other GLP-1A, such as semaglutide and exenatide. ${ }^{44,45}$ Another GLP-1A, dulaglutide, showed positive outcomes in two studies. It reduced the predefined composite renal endpoint and had a significantly lower incidence rate of macroalbuminuria than the placebo in the REWIND trial.-

${ }^{33}$ It also reduced the decline of GFR following 52 weeks of therapy in Tuttle et al study. ${ }^{34}$ Similar positive findings were reported with another two GLP-1As, semaglutide and exenatide, in which both medications reduced the incidence and progression of DKD in patients with T2DM. ${ }^{35,36}$ In the ELIXA trial, ${ }^{38}$ lixisenatide, a GLP-1A, was compared to placebo and found to reduce the progression of ACR in macroalbuminuric patients. It also had a lower risk for new-onset macroalbuminuria after adjusting for HbA1c. In addition to their effect in lowering blood glucose, GLP-1As reduce blood pressure, insulin levels, and weight loss. With the natriuretic effect of GLP-1As, these factors may account for their beneficial effect on the renal system. ${ }^{53}$ Moreover, GLP-1As have been reported to suppress kidney fibrosis. ${ }^{54}$ Although these findings show great promise for diabetic patients, the ability of this group of ADD to retard ESRD progression is yet to be explored. $^{54}$

Many other oral antidiabetic classes had also been studied in some of the included articles such as pioglitazone, which is classified under the class of thiazolidinediones that act by improving the sensitivity of insulin receptors while also reducing the production of glucose by the liver. Chen et al found out that patients taking pioglitazone showed no significant differences in terms of GFR and ACR compared to a group of patients that took acarbose, an $\alpha$-glucosidase inhibitor, that acts by slowing down the breakdown of carbohydrates in the intestine ${ }^{39}$ Further studies are required to make a conclusive statement regarding the effects of pioglitazone and also acarbose on the renal system. In a population-based study, the use of sulphonylureas (SU) was linked to a high risk of hypoglycemia among T2D patients with advanced chronic kidney disease (eGFR $\left.<30 \mathrm{~mL} / \mathrm{min} / 1.73 \mathrm{~m}^{2}\right) .{ }^{40}$ The researchers concluded that sulphonylureas should not be prescribed to this group of patients, unless with caution. It is worth to mention here that studies showed that hypoglycemia is associated with cardiac disturbances, increasing the risk for morbidity and mortality. Moreover, CKD is known as an independent risk factor for hypoglycemia; it also intensifies the risk already present in diabetic patients. ${ }^{55,56}$

\section{Recommendations}

Overall, AD pharmacotherapy's potential renoprotective effects were reported at various evidence levels for SGLT2i, GLP-1A, and DPP4i. Among the incretin-based therapies (DPP4i \& GLP-1A), GLP-1A had relatively more pronounced glucose-independent effects on the renal risk factors among T2DM patients such as decreasing obesity, dyslipidemia, and glomerular hyperfiltration. ${ }^{57}$ Therefore, it appears that more substantial evidence of renoprotective effects has been shown in studies with the use of SGLT2i and GLP-1A compared to those with DPP4i. As per the recent guidelines of the European Association for the study of Diabetes, the nephroprotective effects were reported for three SGLT2i (empagliflozin, canagliflozin, and dapagliflozin) and two GLP-1A (liraglutide and semaglutide). ${ }^{45}$ Also, suppression of GFR decline has been more clearly shown in studies with SGLT2i compared with GLP-1A. Moreover, SGLT2i was associated with the earlier and more consistent incidence of renoprotective effects than GLP-1A, attributed mainly to the underlying hemodynamic changes. ${ }^{58}$ The SGLT2iassociated renoprotective effects have been highlighted as a key mechanism for the reported cardiovascular benefits. ${ }^{43}$ Meanwhile, the potential renoprotective effects of GLP-1A were attributed mainly to its impact on mitigating the progression of atherosclerosis-related vascular disease. ${ }^{52}$ Consistently with our review findings, there are recent recommendations to consider either SGLT2i or GLP-1A as add on to metformin in patients who had mainly underlying atherosclerotic cardiovascular disease, and to consider SGLT2i first, in patients who had underlying chronic kidney disease and reduced heart failure, to 
be followed by GLP-1A if needed. ${ }^{44}$ Furthermore, the most recent American standards of medical care in diabetes have highlighted the role of SGLT2 $\mathrm{i}$ in T2DM patients and DKD, particularly among those with urinary albumin $>300 \mathrm{mg} / \mathrm{g}$ creatinine to mitigate the progression of CKD and cardiovascular events. Whereas GLP-1A is recommended for those $\mathrm{CKD}$ patients at an increased CVD risk to reduce the risk of cardiovascular events and the risk of albuminuria progression. ${ }^{59}$ With close consideration of the bidirectional relationship between kidney and cardiovascular events among patients with diabetes, ${ }^{60}$ those antidiabetic therapies with a favorable impact on renal and cardiovascular outcomes will play a crucial role in reshaping the way of mitigating diabetes complications.

\section{Conclusion}

More than half of the studies included in this review supported the renoprotective effects associated with the use of AD medications, particularly GLP-1A, SGLT2i, and some of the DPP4i. The renal outcomes' impact should be translated into practice with consideration to the baseline renal function and the risk of diabetes complications such as hypoglycemia. There are several recommendations for optimizing the use of SGLT2 $i$ and GLP-1A in the care of diabetes patients at increased risk of renal or cardiovascular events. Furthermore, owing to the differences in the study designs of included studies, there are several recommendations for optimizing the use of SGLT2 $i$ and GLP-1A in the care of diabetic patients who are the increased risk of renal or cardiovascular events. Further analysis and comparison between agents of the same class are also encouraged. Finally, renal outcomes were mostly reported as secondary outcomes or part of the post hoc analysis; it is suggested that future trials setting the extent of nephroprotection as the sole primary outcome will strengthen the evidence of the renal outcomes of various AD pharmacotherapies.

\section{Acknowledgments}

The authors would like to thank Mr. Muhammad Zulkifli for providing support in the initial screening of the included studies.

\section{Disclosure}

The authors report no conflicts of interest for this work.

\section{References}

1. WHO. Global report on diabetes. 2016. Available from: http://www. who.int/about/licensing/copyright_form/index.html. Accessed September 17, 2020.

2. De Boer IH. Kidney disease and related findings in the diabetes control and complications trial/epidemiology of diabetes interventions and complications study. Diabetes Care. 2014;37(1):24-30. doi:10.2337/dc13-2113

3. Gross JL, De Azevedo MJ, Silveiro SP, et al. Diabetic nephropathy: diagnosis, prevention, and treatment. Diabetes Care. 2005;28:176188. doi:10.2337/diacare.28.1.164

4. Williams DM, Nawaz A, Evans M. Renal outcomes in type 2 diabetes: a review of cardiovascular and renal outcome trials. Diabetes Ther. 2020;11(2):369-386. doi:10.1007/s13300-019-00747-3

5. Buse JB, Wexler DJ, Tsapas A, et al. 2019 update to: management of hyperglycemia in type 2 diabetes, 2018. A consensus report by the American diabetes association (ADA) and the European association for the study of diabetes (EASD). Diabetes Care. 2020;43(2):487493. doi: $10.2337 /$ dci19-0066

6. Sung K-C, Ryu S, Lee J-Y, et al. Urine albumin/creatinine ratio below $30 \mathrm{mg} / \mathrm{g}$ is a predictor of incident hypertension and cardiovascular mortality. Am Hear Assoc. 2016;5(9). doi:10.1161/JAHA.116.003245

7. Gerstein HC, Mann JFE, Yi Q, et al. Albuminuria and risk of cardiovascular events, death, and heart failure in diabetic and nondiabetic individuals. $J$ Am Med Assoc. 2001;286(4):421-426. doi:10.1001/jama.286.4.421

8. Zoungas S, Arima H, Gerstein HC, et al. Effects of intensive glucose control on microvascular outcomes in patients with type 2 diabetes: a meta-analysis of individual participant data from randomised controlled trials. Lancet Diabetes Endocrinol. 2017;5(6):431-437. doi:10.1016/S2213-8587(17)30104-3

9. Bell S, Farran B, McGurnaghan S, et al. Risk of acute kidney injury and survival in patients treated with Metformin: an observational cohort study. BMC Nephrol. 2017;18(1):163. doi:10.1186/s12882-017-0579-5

10. Christiansen CF, Ehrenstein V, Heide-Jørgensen U, et al. Metformin initiation and renal impairment: a cohort study in Denmark and the UK. BMJ Open. 2015;5(9):e008531. doi:10.1136/bmjopen-2015-008531

11. Ekström N, Schiöler L, Svensson AM, et al. Effectiveness and safety of metformin in 51675 patients with type 2 diabetes and different levels of renal function: a cohort study from the Swedish national diabetes register. BMJ Open. 2012;2(4):e001076. doi:10.1136/bmjopen-2012-001076

12. Mariano F, Pozzato M, Inguaggiato $\mathrm{P}$, et al. Metformin-associated lactic acidosis undergoing renal replacement therapy in intensive care units: a five-million population-based study in the North-West of Italy. Blood Purif. 2017;44(3):198-205. doi:10.1159/000471917

13. Heerspink HJL, Karasik A, Thuresson M, et al. Kidney outcomes associated with use of SGLT2 inhibitors in real-world clinical practice (CVD-REAL 3): a multinational observational cohort study. Lancet Diabetes Endocrinol. 2020;8(1):27-35. doi:10.1016/S22138587(19)30384-5

14. Mosenzon O, Wiviott SD, Cahn A, et al. Effects of dapagliflozin on development and progression of kidney disease in patients with type 2 diabetes: an analysis from the DECLARE-TIMI 58 randomised trial. Lancet Diabetes Endocrinol. 2019;7(8):606-617. doi:10.1016/ S2213-8587(19)30180-9

15. Fioretto P, Stefansson BV, Johnsson E, Cain VA, Sjöström CD. Dapagliflozin reduces albuminuria over 2 years in patients with type 2 diabetes mellitus and renal impairment. Diabetologia. 2016;59(9):2036-2039. doi:10.1007/s00125-016-4017-1

16. Fioretto P, Del Prato S, Buse JB, et al. Efficacy and safety of dapagliflozin in patients with type 2 diabetes and moderate renal impairment (chronic kidney disease stage 3A): the DERIVE study. Diabetes, Obes Metab. 2018;20(11):2532-2540. doi:10.1111/ dom.13413 
17. Kadowaki T, Nangaku M, Hantel S, et al. Empagliflozin and kidney outcomes in Asian patients with type 2 diabetes and established cardiovascular disease: results from the EMPA-REG OUTCOME ${ }^{\circledR}$ trial. J Diabetes Investig. 2019;10(3):760-770. doi:10.1111/jdi.12971

18. Kashiwagi A, Takahashi H, Ishikawa H, et al. A randomized, doubleblind, placebo-controlled study on long-term efficacy and safety of ipragliflozin treatment in patients with type 2 diabetes mellitus and renal impairment: results of the long-term ASP1941 safety evaluation in patients with type 2 dia. Diabetes, Obes Metab. 2015;17(2):152160. doi:10.1111/dom.12403

19. Wanner C, Inzucchi SE, Lachin JM, et al. Empagliflozin and progression of kidney disease in type 2 diabetes. N Engl J Med. 2016;375 (4):323-334. doi:10.1056/NEJMoa1515920

20. Lambers Heerspink HJ, De Zeeuw D, Wie L, Leslie B, List J. Dapagliflozin a glucose-regulating drug with diuretic properties in subjects with type 2 diabetes. Diabetes, Obes Metab. 2013;15 (9):853-862. doi:10.1111/dom.12127

21. Nadkarni GN, Ferrandino R, Chang A, et al. Acute kidney injury in patients on SGLT2 inhibitors: a propensity-matched analysis. Diabetes Care. 2017;40(11):1479-1485. doi:10.2337/dc17-1011

22. Mahaffey KW, Jardine MJ, Bompoint S, et al. Canagliflozin and cardiovascular and renal outcomes in type 2 diabetes mellitus and chronic kidney disease in primary and secondary cardiovascular prevention groups: results from the randomized CREDENCE trial. Circulation. 2019;140(9):739-750. doi:10.1161/ CIRCULATIONAHA.119.042007

23. Perkovic V, de Zeeuw D, Mahaffey KW, et al. Canagliflozin and renal outcomes in type 2 diabetes: results from the CANVAS program randomised clinical trials. Lancet Diabetes Endocrinol. 2018;6 (9):691-704. doi:10.1016/S2213-8587(18)30141-4

24. Yamout H, Perkovic V, Davies M, et al. Efficacy and safety of canagliflozin in patients with type 2 diabetes and stage 3 nephropathy. Am J Nephrol. 2014;40(1):64-74. doi:10.1159/000364909

25. Cherney DZI, Heerspink HJL, Frederich R, et al. Effects of ertugliflozin on renal function over 104 weeks of treatment: a post hoc analysis of two randomised controlled trials. Diabetologia. 2020;63 (6):1128-1140. doi:10.1007/s00125-020-05133-4

26. Rosenstock J, Perkovic V, Johansen OE, et al. Effect of linagliptin vs placebo on major cardiovascular events in adults with type 2 diabetes and high cardiovascular and renal risk: the CARMELINA randomized clinical trial. JAMA - J Am Med Assoc. 2019;321(1):69-79. doi:10.1001/jama.2018.18269

27. Groop PH, Cooper ME, Perkovic V, et al. Linagliptin and its effects on hyperglycaemia and albuminuria in patients with type 2 diabetes and renal dysfunction: the randomized MARLINA-T2D trial. Diabetes, Obes Metab. 2017;19(11):1610-1619. doi:10.1111/dom.13041

28. Kim YG, Byun J, Yoon D, et al. Renal protective effect of DPP-4 inhibitors in type 2 diabetes mellitus patients: a cohort study. $J$ Diabetes Res. 2016;2016:1-9. doi:10.1155/2016/1423191

29. Mosenzon O, Leibowitz G, Bhatt DL, et al. Effect of saxagliptin on renal outcomes in the SAVOR-TIMI 53 trial. Diabetes Care. 2017;40 (1):69-76. doi:10.2337/dc16-0621

30. Scott R, Morgan J, Zimmer Z, et al. A randomized clinical trial of the efficacy and safety of sitagliptin compared with dapagliflozin in patients with type 2 diabetes mellitus and mild renal insufficiency: the CompoSIT-R study. Diabetes, Obes Metab. 2018;20(12):28762884. doi:10.1111/dom.13473

31. von Eynatten M, Gong Y, Emser A, Woerle HJ. Efficacy and safety of linagliptin in type 2 diabetes subjects at high risk for renal and cardiovascular disease: a pooled analysis of six Phase III clinical trials. Cardiovasc Diabetol. 2013;12(1):1-9. doi:10.1186/14752840-12-60

32. Cornel JH, Bakris GL, Stevens SR, et al. Effect of sitagliptin on kidney function and respective cardiovascular outcomes in type 2 diabetes: outcomes from TECOS. Diabetes Care. 2016;39(12):23042310. doi:10.2337/dc16-1415
33. Gerstein HC, Colhoun HM, Dagenais GR, et al. Dulaglutide and renal outcomes in type 2 diabetes: an exploratory analysis of the REWIND randomised, placebo-controlled trial. Lancet. 2019;394 (10193):131-138. doi:10.1016/S0140-6736(19)31150-X

34. Tuttle KR, Lakshmanan MC, Rayner B, et al. Dulaglutide versus insulin glargine in patients with type 2 diabetes and moderate-tosevere chronic kidney disease (AWARD-7): a multicentre, open-label, randomised trial. Lancet Diabetes Endocrinol. 2018;6(8):605-617. doi:10.1016/S2213-8587(18)30104-9

35. Marso SP, Bain SC, Consoli A, et al. Semaglutide and cardiovascular outcomes in patients with type 2 diabetes. $N$ Engl J Med. 2016;375 (19):1834-1844. doi:10.1056/NEJMoa1607141

36. Bethel MA, Mentz RJ, Merrill P, et al. Renal outcomes in the EXenatide study of cardiovascular event lowering (EXSCEL). Diabetes. 2019;67(Supplement 1):522. doi:10.2337/db18-522-p

37. Mann JFE, Ørsted DD, Brown-Frandsen K, et al. Liraglutide and renal outcomes in type 2 diabetes. N Engl J Med. 2017;377(9):839848. doi:10.1056/NEJMoa1616011

38. Muskiet MHA, Tonneijck L, Huang Y, et al. Lixisenatide and renal outcomes in patients with type 2 diabetes and acute coronary syndrome: an exploratory analysis of the ELIXA randomised, placebocontrolled trial. Lancet Diabetes Endocrinol. 2018;6(11):859-869. doi:10.1016/S2213-8587(18)30268-7

39. Chen YH, Tarng DC, Chen HS. Renal outcomes of pioglitazone compared with acarbose in diabetic patients: a randomized controlled study. PLoS One. 2016;11:11. doi:10.1371/journal.pone.0165750

40. Van Dalem J, Brouwers MCGJ, Stehouwer CDA, et al. Risk of hypoglycaemia in users of sulphonylureas compared with metformin in relation to renal function and sulphonylurea metabolite group: population based cohort study. BMJ. 2016;354. doi:10.1136/bmj. i3625

41. Hsu WH, Hsiao PJ, Lin PC, Chen SC, Lee MY, Shin SJ. Effect of metformin on kidney function in patients with type 2 diabetes mellitus and moderate chronic kidney disease. Oncotarget. 2018;9 (4):5416-5423. doi:10.18632/oncotarget.23387

42. Fioretto P, Zambon A, Rossato M, Busetto L, Vettor R. SGLT2 inhibitors and the diabetic kidney. Diabetes Care. 2016;39(August): S165-S171. doi:10.2337/dcS15-3006

43. Margonato D, Galati G, Mazzetti S, et al. Renal protection: a leading mechanism for cardiovascular benefit in patients treated with SGLT2 inhibitors. Heart Fail Rev. 2020:1-9. doi:10.1007/s10741-02010024-2

44. Sarafidis P, Ferro CJ, Morales E, et al. SGLT-2 inhibitors and GLP-1 receptor agonists for nephroprotection and cardioprotection in patients with diabetes mellitus and chronic kidney disease. A consensus statement by the EURECA-m and the DIABESITY working groups of the ERA-EDTA. Nephrol Dial Transplant. 2019;34 (2):208-230. doi:10.1093/ndt/gfy407

45. Cosentino F, Grant PJ, Aboyans V, et al. 2019 ESC Guidelines on diabetes, pre-diabetes, and cardiovascular diseases developed in collaboration with the EASD. Eur Heart J. 2020;41(2):255-323. doi:10.1093/eurheartj/ehz486

46. Cahn A, Melzer-Cohen C, Pollack R, Chodick G, Shalev V. Acute renal outcomes with sodium-glucose co-transporter-2 inhibitors: realworld data analysis. Diabetes, Obes Metab. 2019;21(2):340-348. doi:10.1111/dom.13532

47. Elnaem MH, Azam FM, Akkawi ME. Cardiovascular outcomes and the use of oral antidiabetic drugs: a review of current evidence from observational studies. IIUM Med J Malaysia. 2020;19(3):100-110. doi:10.31436/imjm.v19i3.1671

48. Coppolino G, Leporini C, Rivoli L, et al. Exploring the effects of DPP-4 inhibitors on the kidney from the bench to clinical trials. Pharmacol Res. 2018;129:274-294. doi:10.1016/j.phrs.2017.12.001

49. Kanasaki K. The role of renal dipeptidyl peptidase-4 in kidney disease: renal effects of dipeptidyl peptidase-4 inhibitors with a focus on linagliptin. Clin Sci. 2018;132(4):489-507. doi:10.1042/CS20180031 
50. Lovshin JA, Rajasekeran H, Lytvyn Y, et al. Dipeptidyl peptidase 4 inhibition stimulates distal tubular natriuresis and increases in circulating sdf-1a1-67 in patients with type 2 diabetes. Diabetes Care. 2017;40(8):1073-1081. doi:10.2337/dc17-0061

51. Preston RA. Renoprotective effects of antihypertensive drugs. Am J Hypertens. 1999;12(1SUPPL. 1):19S-32S. doi:10.1016/S0895-7061 (98)00210-6

52. Ussher JR, Drucker DJ. Cardiovascular actions of incretin-based therapies. Circ Res. 2014;114(11):1788-1803. doi:10.1161/ CIRCRESAHA.114.301958

53. Greco EV, Russo G, Giandalia A, Viazzi F, Pontremoli R, De Cosmo S. GLP-1 receptor agonists and kidney protection. Med. 2019;55 (6):233. doi:10.3390/medicina55060233

54. Kawanami D, Takashi Y. GLP-1 receptor agonists in diabetic kidney disease: from clinical outcomes to mechanisms. Front Pharmacol. 2020;11. doi:10.3389/fphar.2020.00967

55. Moen MF, Zhan M, Hsu VD, et al. Frequency of hypoglycemia and its significance in chronic kidney disease. Clin J Am Soc Nephrol. 2009;4(6):1121-1127. doi:10.2215/CJN.00800209
56. Alsahli M, Gerich J. Hypoglycemia in patients with diabetes and renal disease. J Clin Med. 2015;4(5):948-964. doi:10.3390/ jcm4050948

57. Muskiet MHA, Tonneijck L, Smits MM, et al. GLP-1 and the kidney: from physiology to pharmacology and outcomes in diabetes. Nat Rev Nephrol. 2017;13(10):605-628. doi:10.1038/nrneph.2017.123

58. Marso SP, Daniels GH, Frandsen KB, et al. Liraglutide and cardiovascular outcomes in type 2 diabetes. $N$ Engl J Med. 2016;375 (4):311-322. doi:10.1056/NEJMoa1603827

59. American Diabetes Association. Standards of medical care in diabetes-2020. Diabetes Care. 2020;43(1). doi:10.2337/diacare.29.02.06.dc05-1593

60. Berbari AE. Links between chronic kidney disease and cardiovascular disease: a bidirectional relationship. In: Cardiorenal Syndrome: Mechanisms, Risk and Treatment. Springer Milan; 2010:3-14. doi:10.1007/978-88-470-1463-3_1
International Journal of General Medicine

\section{Publish your work in this journal}

The International Journal of General Medicine is an international, peer-reviewed open-access journal that focuses on general and internal medicine, pathogenesis, epidemiology, diagnosis, monitoring and treatment protocols. The journal is characterized by the rapid reporting of reviews, original research and clinical studies
Dovepress

across all disease areas. The manuscript management system is completely online and includes a very quick and fair peer-review system, which is all easy to use. Visit http://www.dovepress.com/ testimonials.php to read real quotes from published authors. 